\title{
Vaginal Rupture and Evisceration in a Patient With Chronic Rectocele: A Case Report and Literature Review
}

\author{
Jazmín M. Cole ${ }^{\mathrm{a}, \mathrm{c}}$, Paulette I. Abbas ${ }^{\mathrm{a}}$, Aparna Kamat ${ }^{\mathrm{b}}$, Michele Curtis ${ }^{\mathrm{b}}$, Daniel J. Bonville
}

\begin{abstract}
While uterine prolapse is not uncommon in postmenopausal women, vaginal rupture and evisceration of small bowel in a patient without prior abdominal or pelvic surgery is a rare entity. We present a case of a 79-year-old woman with a prolonged history of uterine prolapse and rectocele who presented with vaginal rupture with subsequent evisceration and incarceration of her small bowel mandating emergent surgery.
\end{abstract}

Keywords: Uterine prolapse; Enterocele; Vaginal rupture; Evisceration; Gynecology; General surgery

\section{Introduction}

While uterine prolapse is relatively common in elderly women, occurring in up to half of women over the age of 50, the incidence of evisceration from the prolapse is low. Vaginal evisceration occurs when abdominal contents herniate through a defect or rupture in the vaginal wall [1] and only one-third of patients with evisceration present with visibly incarcerated bowel [2]. Most reported cases of vaginal evisceration that are reported are secondary to trauma or dehiscence of pelvic floor closures. We present a rare case of a woman who not only presented with grossly incarcerated small bowel from a vaginal evisceration, but was secondary to a prolonged history of uterine prolapse in the absence of previous pelvic surgery.

\section{Case Report}

A 79-year-old postmenopausal woman with past medical

Manuscript submitted October 17, 2019, accepted November 5, 2019

a Department of Surgery, Houston Methodist Hospital, Houston, TX 77021, USA

bDepartment of Obstetrics and Gynecology, Houston Methodist Hospital, Houston, TX 77021, USA

${ }^{\mathrm{c} C o r r e s p o n d i n g ~ A u t h o r: ~ J a z m i n ~ M . ~ C o l e, ~ D e p a r t m e n t ~ o f ~ S u r g e r y, ~ H o u s t o n ~}$ Methodist Hospital, 6550 Fannin Street, Smith 1661, Houston, TX 77030, USA. Email: jmcole@houstonmethodist.org

doi: https://doi.org/10.14740/jcs393 history of hypothyroidism, hypertension, diabetes, and cholecystitis status post history of open cholecystectomy, with no major pelvic surgical history, presented to an outside hospital with concern for an abnormal vaginal protrusion. She had three vaginal births in the past, with the most recent one being over 30 years ago. Reportedly, the patient had a 7-year history of uterine prolapse for which she was left untreated for various reasons. She subsequently described a persistent posterior "bulge" to her uterus, consistent with a rectocele, which was stable over the past few years. However, on the morning of admission, the patient noticed that the rectocele became larger after she had a bowel movement. She attempted to reduce the mass, however, noticed that the protrusion grew in size after the attempted manual reduction. Throughout the day, the patient became progressively nauseated and she decided to present to an outside hospital for evaluation.

While at the outside hospital, the patient grew increasingly nauseated and vomited. The emergency room physician evaluated her and grew concerned that there was a probable loop of bowel that had eviscerated and become incarcerated. For this reason, she was transferred to our institution for higher level of care.

Upon transfer, the patient was noted to have a significant amount of abdominal and pelvic discomfort and increased retching. She noted that she passed gas while at the outside facility and had a bowel movement earlier that morning. The patient was hemodynamically stable. Examination revealed a prolapsed uterus, everted vagina with mucosal thickening and ulceration, and loops of eviscerated small bowel with an area of congestion and duskiness, concerning for ischemia (Fig. 1). Additionally, the patient was noted to have two large reducible, non-tender ventral hernias, one at the umbilicus and the other at the lateral aspect of the incision from her previous open cholecystectomy. Given the appearance of the bowel with her symptoms of partial obstruction, it was determined that the patient would require an emergent operation. A nasogastric tube was placed for decompression, intravenous access was obtained to initiate crystalloid fluid for volume resuscitation, and the gynecology service was consulted for assistance. The patient was taken to the operating room that night.

In the operating room, the patient underwent an exploratory laparotomy. There were minimal adhesions, which were lysed. The uterus and eviscerated bowel were then inverted by the gynecologist exerting transvaginal pressure on the uterus and bowel while the general surgeons concomitantly retracted the bowel intra-abdominally. After successful reduction of contents, the loop of eviscerated small bowel was evaluated 


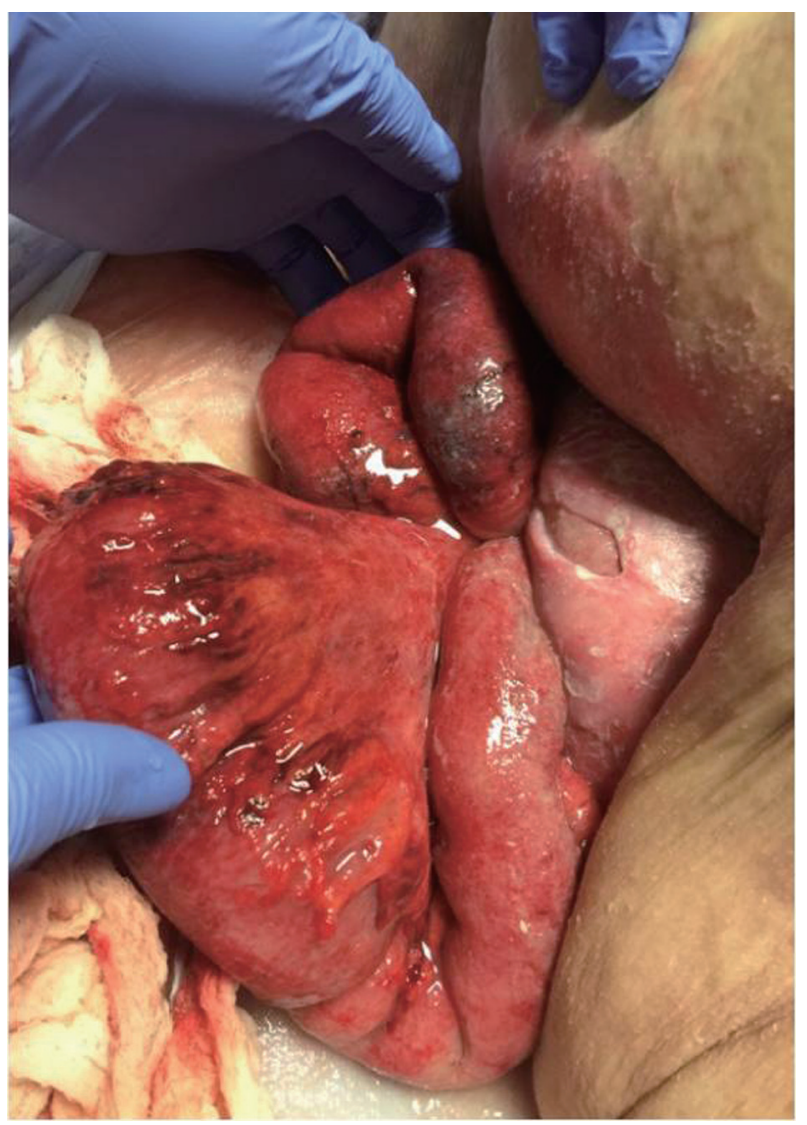

Figure 1. Physical exam findings on presentation. The photo shows eviscerated loops of small bowel with areas of duskiness and congestion concerning for ischemia.

and noted to be congested and edematous but without signs of ischemia or necrosis. The pelvic organs were then addressed by the gynecology oncology team and a total abdominal hysterectomy with bilateral salpingo-oophorectomy was performed with primary closure of the vaginal cuff using interrupted figure-of-eight Vicryl sutures. A defect in the posterior vaginal cul-de-sac at the pouch of Douglas was identified as the site of evisceration (Fig. 2) and this was closed primarily with running \#1 polydioxanone (PDS). The edges of the vaginal disruption were suspended to the vaginal cuff. The ureters and rectum were inspected and noted to be intact and free from injury. At this point, pelvic reconstruction with mesh was felt to be high-risk given the concern for contamination and infection. It was determined that the patient would best benefit from a delayed repair of the pelvic floor, potentially through a transvaginal approach. The abdomen was then closed with excision of the ventral hernia sacs and primary repair of the defects.

The patient was transferred to the intensive care unit (ICU) for monitoring. Her postoperative course was without complications and she was transferred out of the ICU on postoperative day 3 . She was ultimately discharged to a skilled nursing facility on postoperative day 10 with instructions to follow-up with gynecology service in 2 weeks to discuss elective repair. The patient also developed urinary incontinence during her hospital course secondary to the weakened pelvic floor and

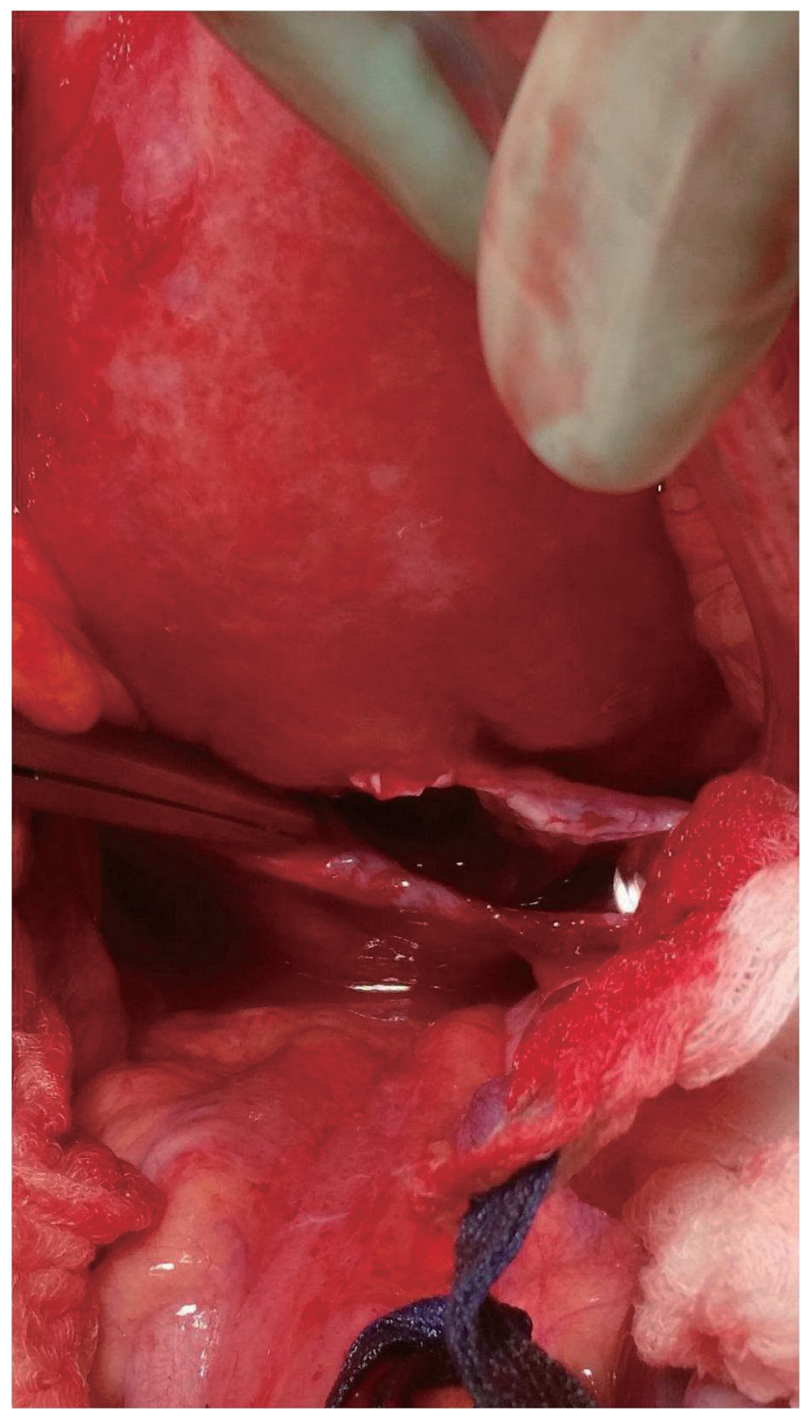

Figure 2. Intraoperative photo showing a defect in the posterior vaginal cul-de-sac at the pouch of Douglas. This was identified as the site of evisceration.

was referred to the urogynecology service as an outpatient.

\section{Discussion}

In our case presentation, the patient was post-menopausal with a history of multiple vaginal deliveries and chronic rectocele, all of which caused weakening of the vaginal tissue. Straining with defecation per the patient's presentation likely increased intrapelvic pressure enough to cause spontaneous vaginal rupture outside of the setting of prior pelvic surgery or pelvic trauma.

In general, historic and anatomic factors predisposing to vaginal evisceration regardless of menopausal status include prior pelvic surgery, the presence of an enterocele, particularly posterior enterocele, vaginal cuff defects, and coital trauma. Post-menopausal women are at increased risk of rupture due 
to atrophic and relatively weaker vaginal tissue than that in pre-menopausal women. In fact, in a case series presented by Croak et al, the most common patient characteristic among their series of women presenting with vaginal rupture and evisceration was a correlative history of prior pelvic surgery and postmenopausal status. The postmenopausal status was felt to contribute to a higher risk secondary to chronic pelvic prolapse and prior pelvic surgery [2]. Additionally, straining with defecation or coughing may also place a patient at additional risk. As expected, prior hysterectomy has also been reported to be significantly associated with vaginal cuff rupture and posterior enterocele rupture, often due to increased intrapelvic pressure on an unhealed vaginal cuff [3].

While increased straining and prolapse have a higher incidence of evisceration, the presence of an enterocele in postmenopausal women further increases their risk of rupture and evisceration. This is likely secondary to the enterocele causing increased pressure on an already weakened atrophic vaginal tissue. As such, vaginal rupture and evisceration frequently occurs at the posterior fornix in affected patients [3, 4], as was seen in our patient. While the presentation and etiology of vaginal evisceration may differ, the management does not significantly differ. Regardless of etiology, prompt management is critical in improving patient outcomes.

Management of vaginal evisceration begins with a thorough history and physical exam as long as patients are stable on presentation. Physical exam should include abdominal exam, focusing on evaluating for peritonitis, as well as examination of the vagina and introitus. Close attention should be paid to inspecting vaginal lacerations or bleeding as well as presence or absence of eviscerated organs. Hur et al recommend a bimanual and speculum exam be performed followed by moist packing gently placed into the vagina in the absence of eviscerated organs. If intra-abdominal organs have eviscerated, these should be protected with saline-soaked wrap en route to emergent surgery [5].

Guttman et al described five initial steps that should be taken when managing a patient with vaginal rupture and evisceration. These include: 1) Stabilizing the patient's hemodynamic status as patients may present shock; 2) Managing patient fluid status and ensuring adequate resuscitation; 3) Preserving eviscerated bowel in saline-soaked wraps; 4) Administering broad-spectrum antibiotics to cover gastrointestinal flora; and 5) Emergent surgical intervention. Both laparoscopic and open approaches have been described; however, the integrity of eviscerated organs should be the surgeon's priority, including resection of nonviable tissue [6]. Similar to our patient, many surgeons elect to perform a delayed pelvic floor repair, particularly in the setting of nonviable vaginal or intraabdominal tissue [7].

\section{Conclusions}

Vaginal rupture and evisceration is a rare but dangerous complication that is often associated with prior pelvic surgery or trauma. However, it can also, even more rarely, occur spontaneously in higher risk patients as presented in our case report. Regardless of the etiology, proper initial resuscitation and prompt surgical management in a multidisciplinary approach may be key in preventing long-term morbidity in these patients. It is important for the management of vaginal rupture and evisceration to be discussed in general surgery literature as general surgeons often play an integral role in delivering multidisciplinary surgical care to these patients.

\section{Acknowledgments}

The authors would like to acknowledge the patient and her family for allowing us to share this case for the purpose of furthering surgical knowledge, as well as the departments of surgery and obstetrics and gynecology for collaborating and supporting the creation of this case report.

\section{Financial Disclosure}

None to declare.

\section{Conflict of Interest}

None to declare.

\section{Informed Consent}

Informed consent for publication was obtained from the patient described in this case report and her family.

\section{Author Contributions}

JMC contributed to literature analysis/search, preparation and editing of the manuscript; PIA contributed to literature analysis/search and editing of the manuscript; DJB contributed to editing of the manuscript, intraoperative photo documentation and data collection; $\mathrm{AK}$ and $\mathrm{MC}$ contributed to intraoperative photo documentation and data collection; All authors contributed to management of the patient.

\section{References}

1. Woo KM, Linden JA, Lowenstein RA, Varghese JC, Burch MA. Subtle vaginal evisceration resulting in small bowel evisceration: a case report. J Emerg Med. 2012;43(2):e125-128.

2. Croak AJ, Gebhart JB, Klingele CJ, Schroeder G, Lee RA, Podratz KC. Characteristics of patients with vaginal rupture and evisceration. Obstet Gynecol. 2004;103(3):572576.

3. Cardosi RJ, Hoffman MS, Roberts WS, Spellacy WN. Vaginal evisceration after hysterectomy in premenopausal women. Obstet Gynecol. 1999;94(5 Pt 2):859.

4. Gheewala U, Agrawal A, Shukla R, Bhatt R, Srivastava 
S. Transvaginal small bowel evisceration in known case of uterine prolapse due to trauma. J Clin Diagn Res. 2015;9(1):PD09-10.

5. Hur HC, Lightfoot M, McMillin MG, Kho KA. Vaginal cuff dehiscence and evisceration: a review of the literature. Curr Opin Obstet Gynecol. 2016;28(4):297-303.
6. Guttman A, Afilalo M. Vaginal evisceration. Am J Emerg Med. 1990;8(2):127-128.

7. Kowalski LD, Seski JC, Timmins PF, Kanbour AI, Kunschner AJ, Kanbour-Shakir A. Vaginal evisceration: presentation and management in postmenopausal women. J Am Coll Surg. 1996;183(3):225-229. 